\title{
Performance Analysis of Adaptive $M$-QAM for Rayleigh Fading Cooperative Systems
}

\author{
T. Nechiporenko, Khoa T. Phan, Chintha Tellambura, \\ Department of Electrical and Computer Engineering, \\ University of Alberta, \\ Edmonton, AB, Canada T6G 2V4, \\ Phone: (780) 492-3332, Fax: (780) 492-1811 \\ Email: nechipor, khoa, chintha@ece.ualberta.ca.
}

\begin{abstract}
The use of constant-power, rate-adaptive $M$-QAM transmission with an amplify-and-forward cooperative system is proposed. The upper bound expressions are derived for the outage probability, achievable spectral efficiency, and error rate performance for the amplify-and-forward cooperative system over both independent and identically distributed (i.i.d.) and non-i.i.d. Rayleigh fading environments. The analysis is based on an accurate upper bound on the total effective signal-tonoise ratio SNR at the destination. Adaptive continuous rate $M$-QAM achieves a capacity that comes within a constant gap of the Shannon capacity of the channel, but adaptive discrete rate $M$-QAM suffers additional performance penalties.
\end{abstract}

\section{INTRODUCTION}

An efficient way of providing diversity in wireless fading networks is through the use of node cooperation for information relaying [1], [2]. In cooperative communications, the source transmits information to the destination not only through a direct-link but also through the use of relays. This so-called "cooperative diversity" can dramatically improve the performance by using the antennas available at the other nodes of the network.

The performance of cooperative communication systems has been analyzed for various system and channel models. The average symbol error rate (SER) of a two-hop cooperative system has been analyzed in [3] for the case of Rayleigh and in [4] for the case of independent, non-identical, Nakagami- $m$ fading channels. The outage performance of the cooperative system with Rayleigh fading operating on a half-duplex mode in the low SNR regime is provided in [5]. Moreover, in [6] and [7] the authors derive the closed-form expressions for the outage probability for the cases of Rayleigh and Nakagami- $m$ channels, respectively, with decode-and-forward relays.

Note that all the aforementioned papers only consider fixed rate and fixed power transmission. However, adaptive wireless transmission techniques are shown to be effective and superior [8]-[11]. Particularly, the transmitter adapts the transmitted power level, symbol rate, constellation size, coding rate/scheme, or any combination of these parameters in response to the changing channel conditions [8]-[11]. This results in an higher average achievable spectral efficiency without having to sacrifice error rate performance.

\author{
Ha H. Nguyen \\ Department of Electrical and Computer Engineering, \\ University of Saskatchewan, \\ Saskatoon, SK, Canada S7N 5A9, \\ Phone: (306) 966-5379, Fax: (306) 966-5407, \\ Email: ha.nguyen@usask.ca
}

Some of the first papers on adaptive transmission are [10], [11], where the performance of several adaptive policies are presented for single-antenna systems over Rayleigh and Nakagami fading channels, respectively, in terms of spectral efficiency and error rate performance. In [9], the same analysis of [10], [11] is extended for diversity reception with multiple receive antennas. Although variable power, variablerate adaptive schemes are shown to be optimum, approaching the capacity of the channel, they have high implementation complexity. Thus, constant power with optimal rate adaptation scheme is a potential candidate for increasing capacity with reduced complexity [12], [13]. In [12], the authors evaluate the corresponding throughput performance and the achievable signal-to-noise ratio (SNR) gain for the optimum mode-switching assisted constant-power adaptive-modulation schemes employing various diversity schemes. Reference [13] proposes and analyzes a rate-adaptive $M$-QAM scheme for orthogonal space time block codes (OSTBC) with outdated, finite-rate feedback.

Motivated by these observations, this paper analyzes the performance of the cooperative systems with constant power $M$-QAM adaptive rate transmission when the average biterror-rate (BER) is constrained to be below some threshold. In particular, we derive the closed-form expressions for the outage probability, achievable spectral efficiency, and BER for the amplify-and-forward cooperative network in both i.i.d. and non-i.i.d. Rayleigh fading environments. Note that these results provide a different perspective on the system implementation and analysis from our previous results [14] where we derive the Shannon capacity for the non-regenerative cooperative system under adaptive transmissions for both i.i.d. and noni.i.d. Rayleigh fading channels.

This paper is organized as follows. Section II presents the channel and system model. Analysis of adaptive $M$-QAM modulation is conducted in Section III. In Section IV the numerical results are presented. Conclusions are given in Section V. 


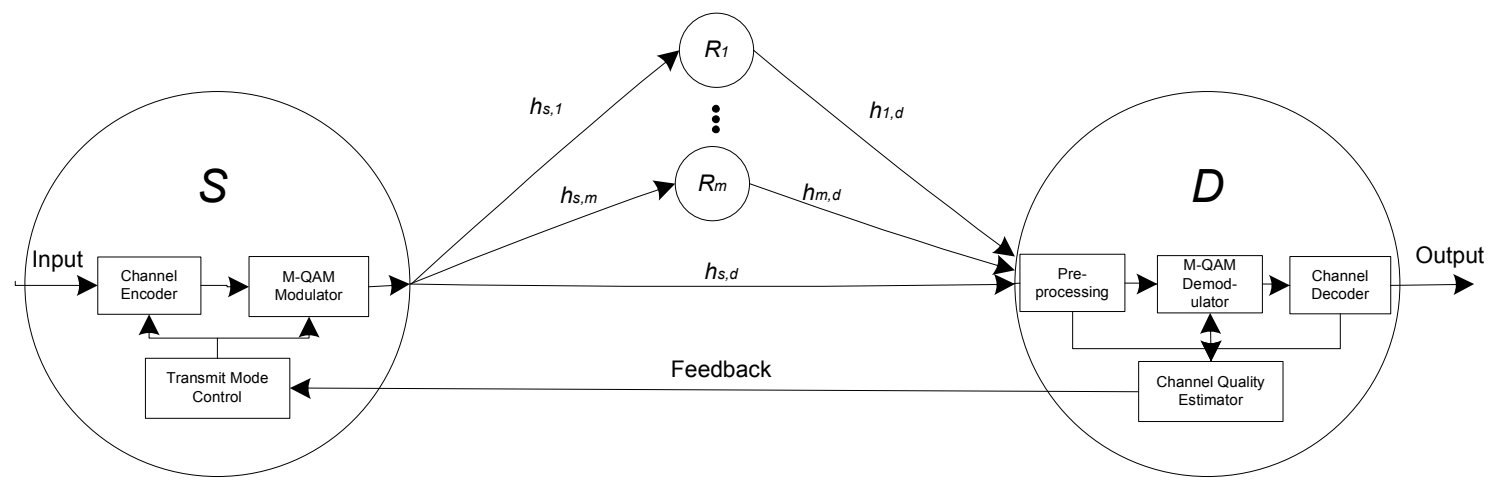

Fig. 1. Cooperative diversity wireless network with adaptive transmission.

\section{Channel And System Model}

\section{A. System Model}

Figure 1 presents the cooperative wireless network, where a source node $S$ communicates with a destination node $D$ via a direct link and through $m$ non-regenerative or amplifyand-forward cooperative relays $R_{i}, i \in\{1,2, \ldots, m\}$. In the first phase of cooperation, the source transmits the signal $x$ to the destination and the relays. The received signals at the destination and at the $i$ th relay respectively are

$$
\begin{gathered}
r_{s, d}=h_{s, d} x+n_{s, d}, \\
r_{s, i}=h_{s, i} x+n_{s, i}
\end{gathered}
$$

where $h_{s, i}$ and $h_{s, d}$ denote the Rayleigh fading coefficients between the source and the $i$ th relay and the source and the destination, respectively. The noise term is denoted at the relays as $n_{s, i}$ and at the destination as $n_{s, d}$ and $n_{i, d}$.

The $i$ th relay amplifies the received signal and transmits to the destination in the second phase of cooperation. However, the second phase of transmission requires $m$ time slots to guarantee orthogonal transmission, and time division multiple access (TDMA) is required [2]. The received signal at the destination from the $i$ th relay is

$$
r_{i, d}=G_{i} h_{i, d} r_{s, i}+n_{i, d}
$$

where $h_{i, d}$ is the Rayleigh fading coefficient between the $i$ th relay and destination and $G_{i}$ is the $i$ th relay amplifier gain, chosen as [2], $G_{i}^{2}=E_{s} /\left(E_{s}\left|h_{s, i}\right|^{2}+N_{0}\right)$ where $E_{s}$ is the average symbol energy, and $N_{0}$ is the noise variance.

At the destination node, using maximum ratio combining (MRC) the total SNR can easily be found to be [2]

$$
\gamma_{\mathrm{tot}}=\gamma_{s, d}+\sum_{i=1}^{m} \frac{\gamma_{s, i} \gamma_{i, d}}{\gamma_{s, i}+\gamma_{i, d}+1}
$$

where $\gamma_{s, i}=\left|h_{s, i}\right|^{2} E_{s} / N_{0}$ is the instantaneous SNR between $S$ and $R_{i}, \gamma_{i, d}=\left|h_{i, d}\right|^{2} E_{s} / N_{0}$ is the instantaneous SNR between $R_{i}$ and $D, \gamma_{s, d}=\left|h_{s, d}\right|^{2} E_{s} / N_{0}$ is the instantaneous SNR between $S$ and $D$.
An upper bound of the total SNR at the destination node can be found as [4], [15]

$$
\gamma_{\text {tot }} \leq \gamma_{s, d}+\sum_{i=1}^{m} \gamma_{i}=\gamma_{u b}
$$

where $\gamma_{i}=\min \left(\gamma_{s, i}, \gamma_{i, d}\right)$. Our subsequent analysis exclusive relies on $\gamma_{u b}$, as this upper-bound has been shown to be quite accurate [4], [14], [15].

\section{B. Probability Distribution Function Derivation}

As $\gamma_{i}$ and $\gamma_{s, d}$ are independent the moment generating function (MGF) of $\gamma_{u b}$ is expressed as

$$
M_{\gamma_{u b}}(s)=M_{\gamma_{s, d}}(s) \prod_{i=1}^{m} M_{\gamma_{i}}(s)
$$

where $M_{\gamma_{s, d}}(s)$ and $M_{\gamma_{i}}(s)$ are the MGF of $\gamma_{s, d}$ and $\gamma_{i}$, respectively, and the MGF is defined as $M_{X}(s)=\mathbf{E}\left\{e^{-s X}\right\}$, $\mathbf{E}\{\cdot\}$ denotes the statistical average over the random variable $X$.

For Rayleigh fading, $\gamma_{s, d}$ is exponentially distributed, thus $M_{\gamma_{s, d}}(s)=\left(1+\bar{\gamma}_{s, d} s\right)^{-1}$, where $\bar{\gamma}_{s, d}=\mathbf{E}\left\{\left|h_{s, d}\right|^{2}\right\} E_{s} / N_{0}$. The MGF of $\gamma_{i}$ is derived via the use of the cumulative distribution function (CDF) of $\gamma_{i}$

$$
F_{\gamma_{i}}(\gamma)=1-P\left(\gamma_{s, i}>\gamma\right) P\left(\gamma_{i, d}>\gamma\right)
$$

To proceed further, consider two different cases of fading channels as follows.

1) The Case of I.I.D. Fading Channels: In this case, the statistic of all links is identical and the average SNR on each link is given by $\bar{\gamma}=\mathbf{E}\left\{\left|h_{s, i}\right|^{2}\right\} E_{s} / N_{0}=\mathbf{E}\left\{\left|h_{i, d}\right|^{2}\right\} E_{s} / N_{0}$. Differentiating (7) the probability density function (pdf) of $\gamma_{i}$ is easily shown to be $p_{\gamma_{i}}(\gamma)=(2 / \bar{\gamma}) e^{-2 \gamma / \bar{\gamma}}$. Then, the MGF can be written as $M_{\gamma_{i}}(s)=(1+0.5 \bar{\gamma} s)^{-1}$. Applying these results into (6) the expression for the MGF of $\gamma_{u b}$ is

$$
M_{\gamma_{u b}}(s)=\left(1+\bar{\gamma}_{s, d} s\right)^{-1}(1+0.5 \bar{\gamma} s)^{-m} .
$$

Using partial fractions, (8) can be rewritten as

$$
M_{\gamma_{u b}}(s)=\beta_{0}\left(1+\bar{\gamma}_{s, d} s\right)^{-1}+\sum_{i=1}^{m} \beta_{i}(1+0.5 \bar{\gamma} s)^{-i}
$$


where

$$
\beta_{0}=\left(1-\frac{\bar{\gamma}}{2 \bar{\gamma}_{s, d}}\right)^{-m}
$$

and

$$
\beta_{i}=\frac{(0.5 \bar{\gamma})^{(i-m)}}{(m-i) !} \frac{\partial^{m-i}}{\partial s^{m-i}}\left[\left(1+\bar{\gamma}_{s, d} s\right)^{-1}\right]_{s=-\frac{1}{(0.5 \bar{\gamma})}} .
$$

Taking the inverse Laplace transform of $M_{\gamma_{u b}}(s)$ in (9), and using the fact that $\mathcal{L}^{-1}\left\{(1+a s)^{-k}\right\}=\frac{1}{(k-1) ! a^{k}} x^{k-1} e^{-\frac{x}{a}}$, the pdf of $\gamma_{u b}$ is as follows:

$$
p_{\gamma_{u b}}(\gamma)=\frac{\beta_{0}}{\bar{\gamma}_{s, d}} e^{-\frac{\gamma}{\bar{\gamma}_{s, d}}}+\sum_{i=1}^{m} \frac{\beta_{i}(0.5 \bar{\gamma})^{-i}}{(i-1) !} \gamma^{i-1} e^{-\frac{\gamma}{(0.5 \bar{\gamma})}} .
$$

2) The Case of Non-I.I.D. Fading Channels: Although the case of i.i.d. fading model simplifies our analysis, in certain environments, it may be more appropriate to consider independent but not identically distributed channels. In practice, it is common that the relays are often not symmetrically placed which causes different fading statistics among the wireless links. Similar to the case of i.i.d. fading, differentiating (7) we obtain the pdf of $\gamma_{i}$ as $p_{\gamma_{i}}(\gamma)=\left(1 / \tau_{i}\right) e^{-\gamma / \tau_{i}}$, where $\tau_{i}=\frac{\bar{\gamma}_{s, i} \bar{\gamma}_{i, d}}{\bar{\gamma}_{s, i}+\bar{\gamma}_{i, d}}, \bar{\gamma}_{s, i}=\mathbf{E}\left\{\left|h_{s, i}\right|^{2}\right\} E_{s} / N_{0}$ and $\bar{\gamma}_{i, d}=$ $\mathbf{E}\left\{\left|h_{i, d}\right|^{2}\right\} E_{s} / N_{0}$. The MGF can then be written as $M_{\gamma_{i}}(s)=$ $\left(1+\tau_{i} s\right)^{-1}$. Applying these results into (6) the MGF of $\gamma_{u b}$ is

$$
M_{\gamma_{u b}}(s)=\left(1+\bar{\gamma}_{s, d} s\right)^{-1} \prod_{i=1}^{m}\left(1+\tau_{i} s\right)^{-1} .
$$

Again, using partial fractions, (13) can be rewritten

$$
M_{\gamma_{u b}}(s)=\widehat{\beta}_{0}\left(1+\gamma_{s, d} s\right)^{-1}+\sum_{i=1}^{m} \widehat{\beta}_{i}\left(1+\tau_{i} s\right)^{-1}
$$

where

$$
\widehat{\beta}_{0}=\prod_{i=1}^{m}\left(1-\frac{\tau_{i}}{\bar{\gamma}_{s, d}}\right)^{-1}
$$

and

$$
\widehat{\beta}_{i}=\left(1-\frac{\bar{\gamma}_{s, d}}{\tau_{i}}\right)^{-1} \prod_{k=1, k \neq i}^{m}\left(1-\frac{\tau_{k}}{\tau_{i}}\right)^{-1}, \quad i=1, \ldots, m .
$$

Taking the inverse Laplace transform of $M_{\gamma_{u b}}(s)$ in (14) gives the pdf of $\gamma_{u b}$ as:

$$
p_{\gamma_{u b}}(\gamma)=\frac{\widehat{\beta}_{0}}{\bar{\gamma}_{s, d}} e^{-\frac{\gamma}{\bar{\gamma}_{s, d}}}+\sum_{i=1}^{m} \frac{\widehat{\beta}_{i}}{\tau_{i}} e^{-\frac{\gamma}{\tau_{i}}}
$$

\section{AdAPTIVE $M$-QAM MOdUlation}

\section{A. Adaptive Scheme}

Among different adaptive transmission schemes, the constant-transmit-power and variable-rate scheme has many practical advantages. The destination needs only to calculate the total SNR and select the appropriate transmission rate and send this information back to the transmitter. This makes adaptive techniques based on adaptive $M$-QAM modulation such as those proposed in [10], [11] viable. For this reason, adaptive $M$-QAM is also considered in this paper. The bit error rate (BER) of a system which implements $M$-QAM modulation over an additive white Gaussian noise (AWGN) channel, with coherent detection and Gray coding can be approximated as [10], [11]

$$
\operatorname{BER}(M, \gamma) \approx 0.2 \exp \left(\frac{3 \gamma}{2(M-1)}\right)
$$

where again $\gamma$ is the received SNR.

It is shown in [11] that the approximate BER (18) is indeed an upper bound on the exact BER for modulation order $M \geq 4$ and for BER $\leq 10^{-2}$. This approximation is used in [10], [11] and in our work due to the fact that it is invertible, which yields nice closed-form solutions for determining the spectral efficiency, outage probability, and average BER. By inverting (18) the spectral efficiency of the continuous-rate $M$-QAM can be approximated as [10], [11]:

$$
\frac{R}{W}=\log _{2} M=\log _{2}\left(1+\frac{3 \gamma}{2 K_{0}}\right)
$$

where $K_{0}=-\ln \left(5 \mathrm{BER}_{0}\right)$ and $\mathrm{BER}_{0}$ is the target BER. The spectral efficiency of (19) is achieved by using the adaptive continuous rate (ACR) $M$-QAM [16]. How ACR $M$-QAM works is the number of bits per symbol is varied depending on the instantaneous effective SNR $\gamma$. However, because of the practical limitation of ACR, adaptive discrete rate (ADR) $M$-QAM with constellation size $M_{n}=2^{n}$ for $n$ a positive integer, is implementable and is worth investigating.

The ADR $M$-QAM scheme performs as follows. The range of the effective received SNR is divided into $N+1$ regions. In each region, a specific constellation of size $M_{n}$ is used. When the fading causes the effective SNR to fall into the $n$th region $(n=0,1, \ldots, N)$, the constellation of size $M_{n}$ is used for transmission. The partitioning of the effective received SNR depends on the desired BER level, $\mathrm{BER}_{0}$. For instance, to have reliable communication which achieves a specific BER target of $\mathrm{BER}_{0}$ using $M_{n}$-QAM, the region boundaries are set to the SNR required to achieve this desired performance, which can easily be shown to be [11]:

$$
\begin{aligned}
& \gamma_{1}=\left[\operatorname{erfc}^{-1}\left(2 \mathrm{BER}_{0}\right)\right]^{2}, \\
& \gamma_{n}=\frac{2}{3} K_{0}\left(2^{n}-1\right) ; \quad n=0,2,3, \ldots, N \\
& \gamma_{N+1}=+\infty
\end{aligned}
$$

where $\operatorname{efrc}^{-1}()$ is the inverse complementary error function. This partition of the region boundaries is provided in [11], but presented here in Fig. 2 for clarity, for a desired BER B $_{0}$ of $10^{-3}$.

\section{B. Outage Probability}

1) The Case of I.I.D. Fading Channels: As no transmission occurs when the received SNR is below the smallest predetermined threshold $\gamma_{1}$, the probability of such an outage event is given by:

$$
\begin{aligned}
P_{\text {out }} & =P\left[\gamma_{u b}<\gamma_{1}\right]=\int_{0}^{\gamma_{1}} p_{\gamma_{u b}}(\gamma) d \gamma \\
& =1-\int_{\gamma_{1}}^{\infty} p_{\gamma_{u b}}(\gamma) d \gamma
\end{aligned}
$$




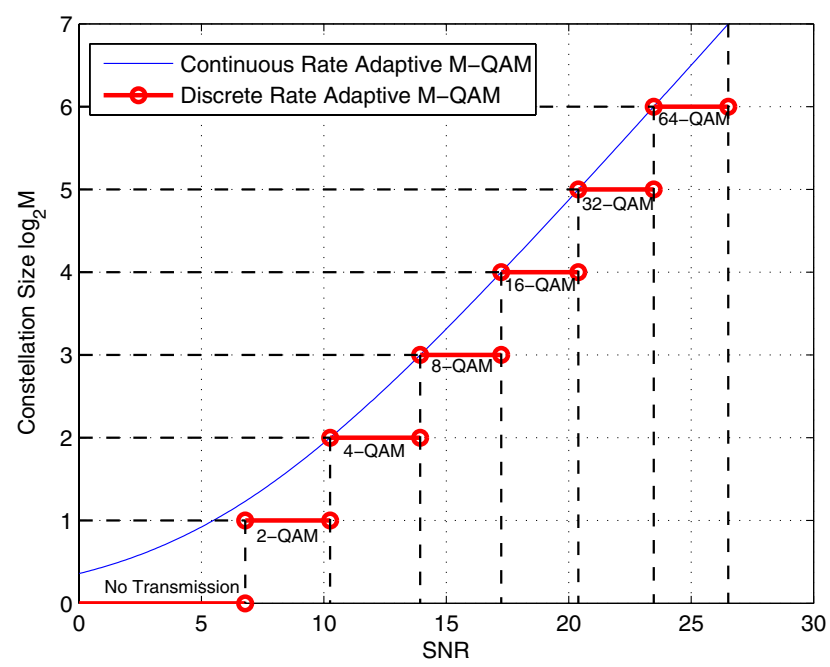

Fig. 2. Constellation size relative to the received SNR (dB).

where

$$
\int_{\gamma_{1}}^{\infty} p_{\gamma_{u b}}(\gamma) d \gamma=\beta_{0} e^{-\frac{\gamma_{1}}{\bar{\gamma}_{s, d}}}+e^{-\frac{2 \gamma_{1}}{\bar{\gamma}}} \sum_{i=1}^{m} \beta_{i} \sum_{k=0}^{i-1} \frac{1}{k !}\left(\frac{2 \gamma_{1}}{\bar{\gamma}}\right)^{k}
$$

is established by using [17, eq. (3.351.2)].

The probability of outage is found by substituting (22) into (21), resulting in

$$
P_{\text {out }}=1-\left[\beta_{0} e^{-\frac{\gamma_{1}}{\gamma_{s, d}}}+e^{-\frac{2 \gamma_{1}}{\bar{\gamma}}} \sum_{i=1}^{m} \beta_{i} \sum_{k=0}^{i-1} \frac{1}{k !}\left(\frac{2 \gamma_{1}}{\bar{\gamma}}\right)^{k}\right]
$$

2) The Case of Non-I.I.D. Fading Channels: In a similar fashion as the i.i.d case, the tail probability is

$$
\int_{\gamma_{1}}^{\infty} p_{\gamma_{u b}}(\gamma) d \gamma=\widehat{\beta}_{0} e^{-\frac{\gamma_{1}}{\gamma_{s, d}}}+\sum_{i=1}^{m} \widehat{\beta}_{i} e^{-\frac{\gamma_{1}}{\tau_{i}}}
$$

Likewise, the probability of outage is found by substituting (24) into (21) to give

$$
P_{\text {out }}=1-\left[\widehat{\beta}_{0} e^{-\frac{\gamma_{1}}{\gamma_{s, d}}}+\sum_{i=1}^{m} \widehat{\beta}_{i} e^{-\frac{\gamma_{1}}{\tau_{i}}}\right] .
$$

\section{Achievable Spectral Efficiency}

1) The Case of I.I.D. Fading Channels: The average spectral efficiency for ACR is found by integrating (19) over the PDF of the received SNR.

$$
R_{\mathrm{acr}}=\frac{B}{(m+1) \ln 2} \int_{0}^{\infty} \ln \left(1+\frac{3 \gamma}{2 K_{0}}\right) p_{\gamma_{u b}}(\gamma) d \gamma
$$

Substituting (12) into (26), and making use of the integral $\mathcal{I}_{n}(\mu)$,

$$
\begin{gathered}
\mathcal{I}_{n}(\mu)=\int_{0}^{\infty} t^{n-1} \ln (1+t) e^{-\mu t} d t, \\
\mu>0 ; \quad n=1,2, \ldots
\end{gathered}
$$

which can be evaluated in a closed-form as in [9, eq. (78)], the closed-form expression for the achievable ACR spectral efficiency $R_{\text {acr }}$ is

$$
\begin{aligned}
R_{\mathrm{acr}}= & \frac{B}{(m+1) \ln 2}\left[\frac{2 K_{0} \beta_{0}}{3 \bar{\gamma}_{s, d}} \mathcal{I}_{1}\left(\frac{2 K_{0}}{3 \bar{\gamma}_{s, d}}\right)\right. \\
& \left.+\sum_{i=1}^{m} \frac{\beta_{i} 4 K_{0}(3 \bar{\gamma})^{-i}}{(i-1) !} \mathcal{I}_{i}\left(\frac{4 K_{0}}{3 \bar{\gamma}}\right)\right] .
\end{aligned}
$$

For the case of ADR the achievable average spectral efficiency is simply the sum of the data rates in each of the partition regions weighted by the probability of occurrence of each region and is given as follows:

$$
R_{\mathrm{adr}}=\frac{B}{(m+1)} \sum_{n=1}^{N} n a_{n},
$$

where $n=\log _{2}\left(M_{n}\right)$ corresponds to the data rate of the $n$th region, $a_{n}$ is the probability that the effective received SNR is in the $n$th partition region and is given as,

$$
a_{n}=\int_{\gamma_{n}}^{\gamma_{n+1}} p_{\gamma_{u b}}(\gamma) d \gamma
$$

which evaluates to

$$
\begin{array}{r}
a_{n}=\beta_{0}\left(e^{-\gamma_{n} / \gamma_{s, d}}-e^{-\gamma_{n+1} / \gamma_{s, d}}\right) \\
+\sum_{i=1}^{m} \beta_{i}\left(\mathcal{P}_{i}\left(\frac{2 \gamma_{n}}{\bar{\gamma}}\right)-\mathcal{P}_{i}\left(\frac{2 \gamma_{n+1}}{\bar{\gamma}}\right)\right)
\end{array}
$$

and where $\mathcal{P}_{i}(\cdot)$ denotes the Poisson distribution, defined as

$$
\mathcal{P}_{i}(\mu)=e^{-\mu} \sum_{j=1}^{i-1} \frac{\mu^{j}}{j !} .
$$

2) The Case of Non-I.I.D. Fading Channels: In a similar manner to the i.i.d fading channel, the closed-form expression for the average ACR spectral efficiency $R_{\text {acr }}$ is

$$
\begin{array}{r}
R_{\mathrm{acr}}=\frac{B}{(m+1) \ln 2}\left[\frac{2 K_{0} \widehat{\beta}_{0}}{3 \bar{\gamma}_{s, d}} \mathcal{I}_{1}\left(\frac{2 K_{0}}{3 \bar{\gamma}_{s, d}}\right)\right. \\
\left.+\sum_{i=1}^{m} \frac{2 K_{0} \widehat{\beta}_{i}}{3 \tau_{i}} \mathcal{I}_{1}\left(\frac{2 K_{0}}{3 \tau_{i}}\right)\right] .
\end{array}
$$

The average ADR spectral efficiency $R_{\mathrm{adr}}$ for the non-i.i.d. fading channel can be found by using (29), where $a_{n}$ is,

$$
\begin{aligned}
a_{n} & =\widehat{\beta}_{0}\left(e^{-\gamma_{n} / \gamma_{s, d}}-e^{-\gamma_{n+1} / \gamma_{s, d}}\right) \\
& +\sum_{i=1}^{m} \widehat{\beta}_{i}\left(e^{-\gamma_{n} / \tau_{i}}-e^{-\gamma_{n+1} / \tau_{i}}\right)
\end{aligned}
$$

\section{Average Bit Error Rate}

1) The Case of I.I.D. Fading Channels: As ACR M-QAM constantly meets the desired BER target, the BER analysis is trivial. However, for the ADR $M$-QAM the discrete partitions of the received SNR results in a conservative average BER. This results in the average $\mathrm{BER}_{\mathrm{adr}}$ always being smaller then 
the target BER. The average $\mathrm{BER}_{\mathrm{adr}}$ can easily be calculated as it is simply the ratio of the average number of bits in error divided by the total average number of transmitted bits,

$$
\mathrm{BER}_{\mathrm{adr}}=\frac{\sum_{n=1}^{N} n \overline{\mathrm{BER}}_{n}}{\sum_{n=1}^{N} n a_{n}},
$$

where

$$
\overline{\operatorname{BER}}_{n}=\int_{\gamma_{n}}^{\gamma_{n+1}} \operatorname{BER}\left(M_{n}, \gamma\right) p_{\gamma_{u b}}(\gamma) d \gamma
$$

By substituting (12) and (18) into (36) the closed-form expression for the average BER for the $n$th region is

$$
\begin{array}{r}
\overline{\operatorname{BER}}_{n}=\frac{0.2 \beta_{0}}{1+\bar{\gamma}_{s, d} b_{n}}\left(e^{-\gamma_{n}\left(\gamma_{s, d}^{-1}+b_{n}\right)}-e^{-\gamma_{n+1}\left(\gamma_{s, d}^{-1}+b_{n+1}\right)}\right) \\
+\sum_{i=1}^{m} \frac{0.2 \beta_{i}}{\left(1+0.5 \bar{\gamma} b_{n}\right)^{i}}\left(\mathcal{P}_{i}\left(\gamma_{n}\left(2 \bar{\gamma}^{-1}+b_{n}\right)\right)\right. \\
\left.-\mathcal{P}_{i}\left(\gamma_{n+1}\left(2 \bar{\gamma}^{-1}+b_{n}\right)\right)\right)
\end{array}
$$

where

$$
b_{n}=\frac{3}{2\left(2^{n}-1\right)} .
$$

2) The Case of Non-I.I.D. Fading Channels: The average $\mathrm{BER}_{\mathrm{adr}}$ for the non-i.i.d fading channel can be found by substituting in $\overline{\mathrm{BER}}_{n}$ into (35), where

$$
\begin{aligned}
& \overline{\mathrm{BER}}_{n}=\frac{0.2 \widehat{\beta}_{0}}{1+\bar{\gamma}_{s, d} b_{n}}\left(e^{-\gamma_{n}\left(\gamma_{s, d}^{-1}+b_{n}\right)}-e^{-\gamma_{n+1}\left(\gamma_{s, d}^{-1}+b_{n}\right)}\right) \\
& +\sum_{i=1}^{m} \frac{0.2 \beta_{i}}{1+b_{n} \tau_{i}}\left(e^{-\gamma_{n}\left(\tau_{i}^{-1}+b_{n}\right)}-e^{-\gamma_{n+1}\left(\tau+i^{-1}+b_{n+1}\right)}\right) .
\end{aligned}
$$

\section{NUMERICAL RESUlTS AND COMPARISONS}

In this section, numerical results for the outage probability, achievable spectral efficiency, and BER analysis for cooperative systems with adaptive $M$-QAM transmissions are presented. For all the numerical results, the i.i.d. Rayleigh fading one relay $(m=1)$, the system model as in [14], is considered. Although such a model is basic, the results of Section III can be used for any number of relays. The effects of path-loss or shadowing can also be taken into account readily. For the case of non-i.i.d. Rayleigh fading, all the numerical results are obtained with two relays $(m=2)$. The average SNR of the links are chosen arbitrarily as in [14] such that they represent a realistic model of a practical cooperative communication system. Specifically, they are as follows: $\bar{\gamma}_{s, 1}=E_{s} / N_{0}, \bar{\gamma}_{s, 2}=0.8 E_{s} / N_{0}, \bar{\gamma}_{1, d}=0.3 E_{s} / N_{0}$, $\bar{\gamma}_{1, d}=0.56 E_{s} / N_{0}$, and $\bar{\gamma}_{s, d}=0.2 E_{s} / N_{0}$.

Fig. 3 and Fig. 4 show the probability of outage (23) and (25) for the $M$-QAM rate adaptation under various target BER levels, for the i.i.d., and non-i.i.d. fading models, respectively. Note that the Monte Carlo simulation results is also plotted to show the accuracy of the upper bound. It is quite clear to see the distinction between the diversity order for the $m=1$ i.i.d. systems and the $m=2$ non-i.i.d. systems. Also as expected,

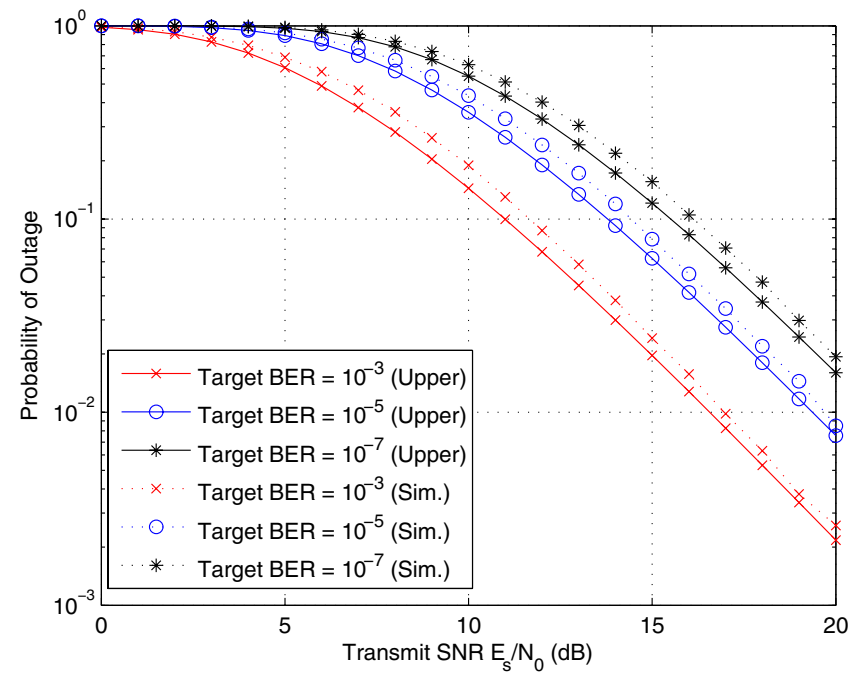

Fig. 3. Outage probability of adaptive schemes with i.i.d. Rayleigh fading channels.

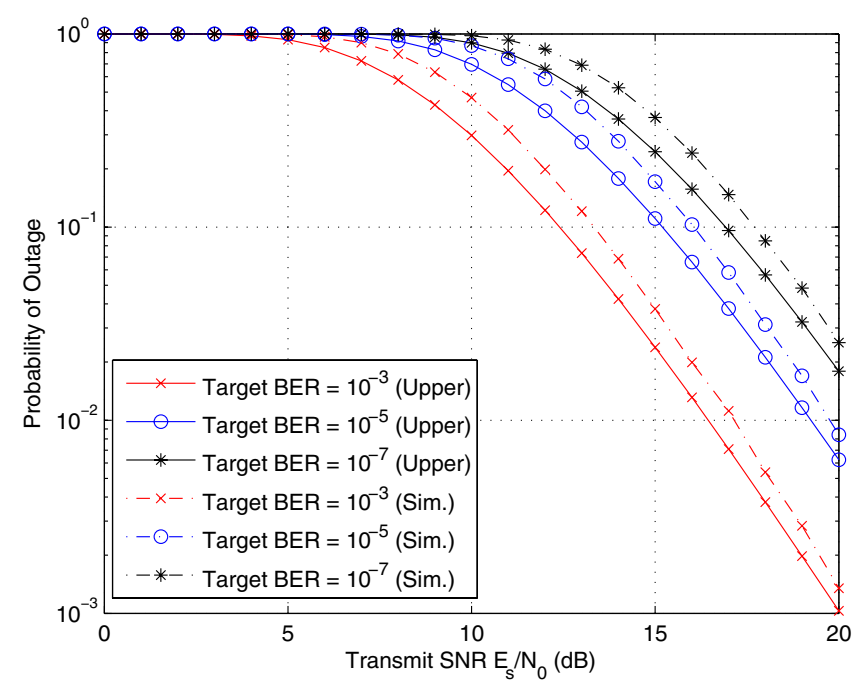

Fig. 4. Outage probability of adaptive schemes with non-i.i.d. Rayleigh fading channels.

the diversity order of each system is independent of the BER target level.

In Fig. 5 the achievable spectral efficiency of ACR $M$ QAM (28) and ADR $M$-QAM (29) for the target BER level of $\mathrm{BER}_{0}=10^{-3}$ for the i.i.d. fading model is plotted. Also plotted for comparison is the Shannon capacity of the optimal rate and constant power cooperative system with i.i.d. Rayleigh fading found in $[14,(32)]$. Furthermore, the Monte Carlo simulation results is plotted. Similarly, in Fig. 6 the achievable spectral efficiency of ACR $M$-QAM (33) and ADR $M$-QAM (29) for the target BER level of $\mathrm{BER}_{0}=10^{-3}$ for the noni.i.d. fading model is plotted and compared with the Shannon capacity of the optimal rate and constant power system [14, (33)]. Fig. 5 and Fig. 6 show that ACR $M$-QAM comes within 
$5 \mathrm{~dB}$ of the Shannon capacity limit. These figures are also similar in the performance of the achievable spectral efficiency of ADR $M$-QAM. For both cases of Rayleigh fading channels, ADR $M$-QAM suffers at least an additional $1.5 \mathrm{~dB}$ compared to the spectral efficiency of ACR $M$-QAM.

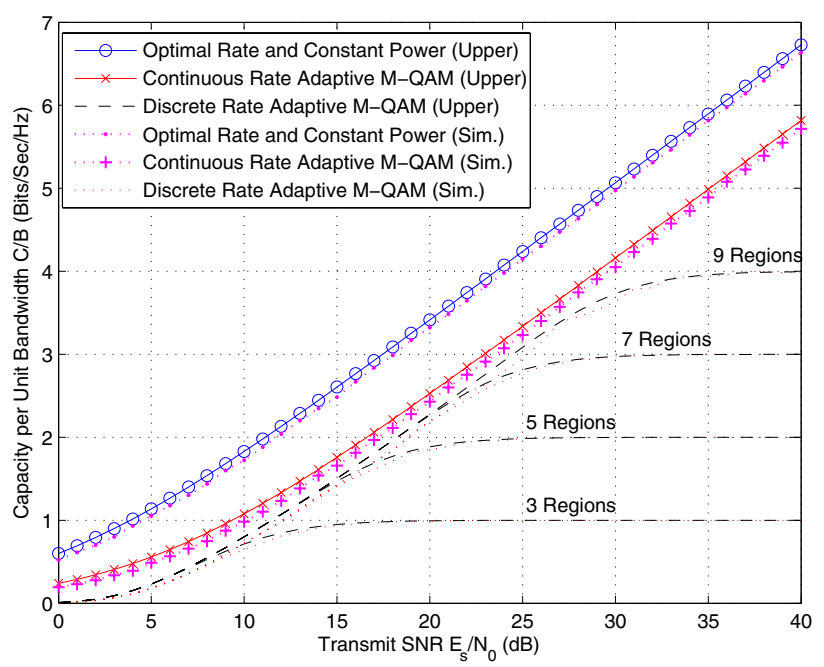

Fig. 5. Achievable rates of adaptive schemes with i.i.d. Rayleigh fading channels.

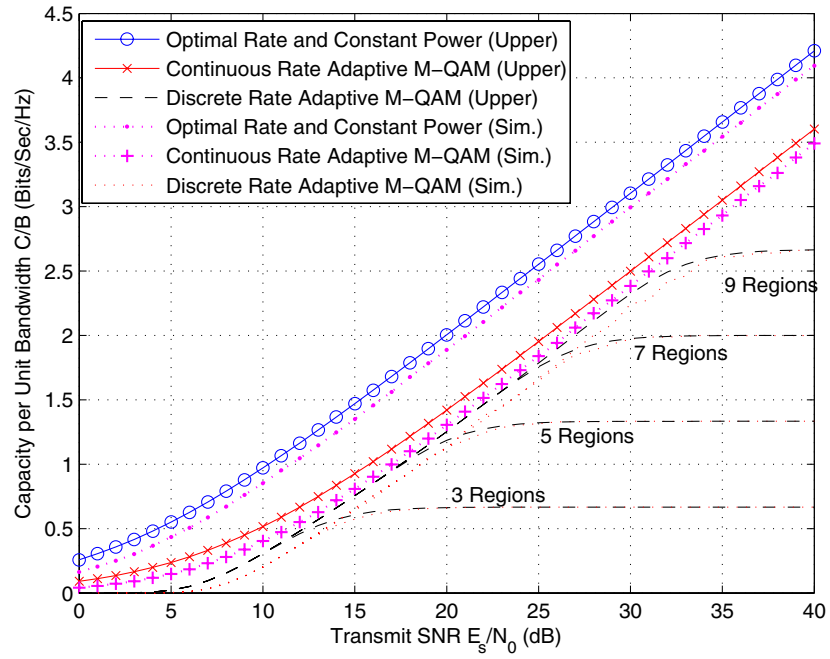

Fig. 6. Achievable rates of adaptive schemes with non-i.i.d. Rayleigh fading channels.

The BER (35) for the ADR $M$-QAM for both i.i.d. and non-i.i.d. Rayleigh fading model is plotted in Fig. 7 and Fig. 8 for the BER target of $\mathrm{BER}_{0}=10^{-3}$. Since ACR $M$-QAM is instantaneous continuous adaptation, it always meets the BER target level. The average BER ADR $M$-QAM is always below the target level, this is because the switching thresholds are chosen such that the instantaneous BER is always guaranteed to be below the BER target level $\mathrm{BER}_{0}$. This results in an inefficient use of spectrum (lower spectral efficiency) in terms of average BER. Although the switching thresholds can be optimized [12], this is not considered in this paper as some applications do require to meet instantaneous BER levels.

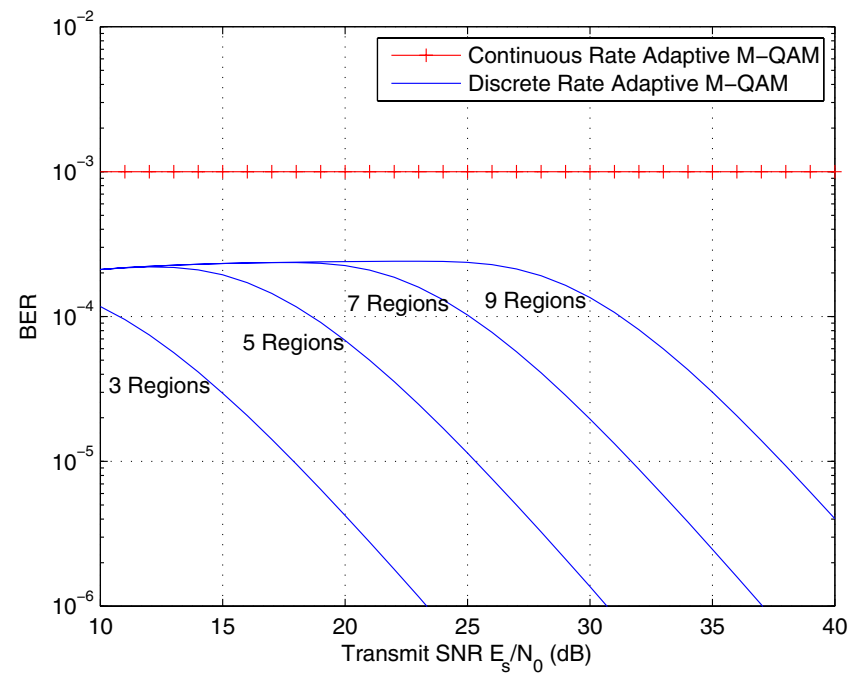

Fig. 7. Average BER for i.i.d. Rayleigh fading channels.

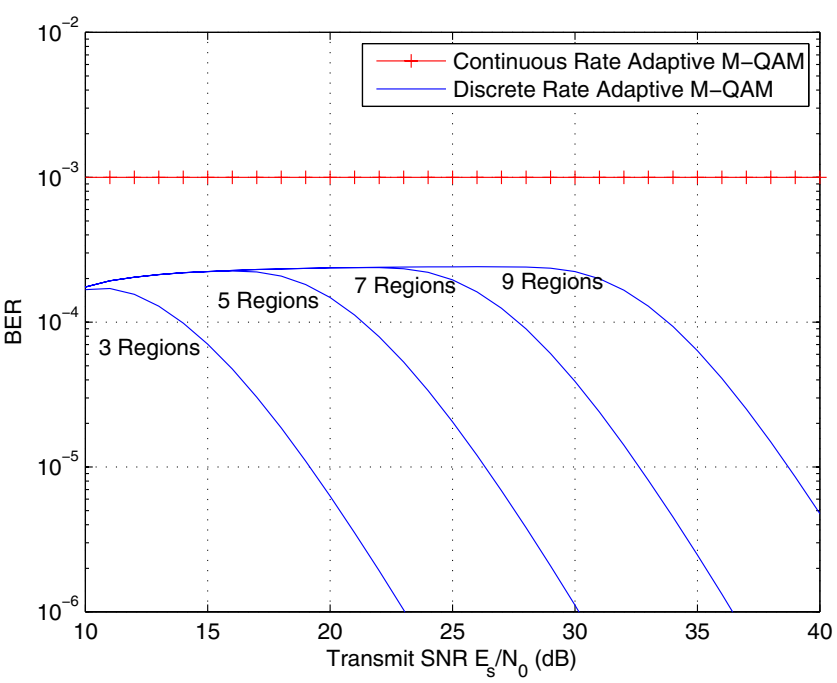

Fig. 8. Average BER for non-i.i.d. Rayleigh fading channels.

\section{CONCLUSION}

This paper investigated adaptive $M$-QAM schemes for i.i.d. and non-i.i.d. Rayleigh fading cooperative channels. Specifically, the outage probability, achievable spectral efficiency, and BER were derived using an upper bound on the effective received SNR. The results indicate that ACR $M$-QAM approaches the Shannon capacity within $5 \mathrm{~dB}$ for both the i.i.d. and non-i.i.d Rayleigh fading models. However, the performance of ADR $M$-QAM results in an additional penalty. Our analysis is based on an upper bound on the 
effective received SNR (5) and on the approximate BER performance of $M$-QAM (18). Monte Carlo simulation is used to show the accuracy regarding the performance of using the effective received SNR bound (5). As well, we are currently investigating the tightness of the approximate BER of $M$ QAM and optimization of the switching thresholds.

\section{REFERENCES}

[1] A. Sendonaris, E. Erkip, and B. Aazhang, "User cooperation diversity part I: system description," IEEE Trans. Commun., vol. 51, pp. 19271938, Nov. 2003.

[2] J. N. Laneman, D. N. C. Tse, and G. W. Wornell, "Cooperative diversity in wireless networks: Efficient protocols and outage behavior," IEEE Trans. Inform. Theory, vol. 50, pp. 3062-3080, Dec. 2004.

[3] M. O. Hasna and M.-S. Alouini, "End-to-end performance of transmission system with relays over Rayleigh-fading channels," IEEE Trans. Wireless Commun., vol. 2, pp. 1126-1131, Nov. 2003.

[4] S. Ikki and M. H. Ahmed, "Performance analysis of cooperative diversity wireless networks over Nakagami- $m$ fading channel," IEEE Commun. Letters, vol. 11, pp. 334-336, July 2007.

[5] A. S. Avestimehr and D. N. C. Tse, "Outage capacity of the fading relay channel in the low-SNR regime," IEEE Trans. Inform. Theory, vol. 53, pp. 1401-1415, Apr. 2007.

[6] N. C. Beaulieu and J. Hu, "A closed-form expression for the outage probability of decode-and-forward relaying in dissimilar Rayleigh fading channels," IEEE Commun. Letters, vol. 10, pp. 813-815, Dec. 2006.

[7] H. A. Suraweera, P. J. Smith, and J. Armstrong, "Outage probability of cooperative relay networks in Nakagami- $m$ fading channels," IEEE Commun. Letters, vol. 10, pp. 834-836, Dec. 2006.

[8] A. J. Goldsmith and P. P. Varaiya, "Capacity of fading channels with channel side information," IEEE Trans. Inform. Theory, vol. 43, pp. 1986-1992, Nov. 1997.

[9] M.-S. Alouini and A. J. Goldsmith, "Capacity of Rayleigh fading channels under different adaptive transmission and diversity-combining techniques," IEEE Trans. Veh. Technol., vol. 48, pp. 1165-1181, July 1999.

[10] A. J. Goldsmith and S.-G. Chua, "Variable-rate variable-power MQAM for fading channels," IEEE Trans. Commun., vol. 45, pp. 1218-1230, Oct. 1997.

[11] M.-S. Alouini and A. J. Goldsmith, "Adaptive modulation over Nakagami fading channels," Wireless Personal Comm., vol. 13, pp. 119-143, 2000.

[12] B. Choi and L. Hanzo, "Optimum mode-switching-assisted constantpower single- and multicarrier adaptive modulation," IEEE Trans. Veh. Technol., vol. 52, pp. 536-560, May 2003.

[13] Y. Ko and C. Tepedelenlioglu, "Orthogonal space-time block coded rateadaptive modulation with outdated feedback," IEEE Trans. on Wireless Commun., vol. 5, pp. 290-295, Feb. 2006.

[14] T. Nechiporenko, K. T. Phan, C. Telllambura, and H. H. Nguyen, "Capacity of Rayleigh fading cooperative systems under adaptive transmission," submitted to IEEE Trans. on Wireless Commun., available online at http://www.ece.ualberta.ca/ nechipor.

[15] P. A. Anghel and M. Kaveh, "Exact symbol error probability of a cooperative network in a Rayleigh-fading environment," IEEE Trans. Wireless Commun., vol. 3, pp. 1416-1421, Sept. 2004

[16] J. Forney, G., R. Gallager, G. Lang, F. Longstaff, and S. Qureshi, "Efficient modulation for band-limited channels," IEEE J. Select. Areas in Commun., vol. 2, pp. 632-647, Sept. 1984.

[17] I. Gradshteyn and I. Ryzhik, Table of Integrals, Series, and Products, Sixth Edition. Academic Press, 2000. 